\title{
Ultra Thin Deployable Reflector Antennas
}

\author{
Lin Tze Tan* \\ University College London, London, WC1E 6BT, UK \\ Sergio Pellegrino ${ }^{\dagger}$ \\ University of Cambridge, Cambridge, CB2 1PZ, UK
}

\begin{abstract}
We have recently proposed a new concept for deployable reflectors consisting of a thin parabolic carbon-fibre-reinforced-plastic (CFRP) shell stiffened along the edge by an elastically collapsible stiffener. Here we show that a stiffener accounting for less than $10 \%$ of the total mass of the reflector increases the stiffness of the softest deformation mode of the deployed reflector by 48 times, and its fundamental natural frequency of vibration by 6.8 times. These values are based on an optimized design of the stiffener, for a $4.6 \mathrm{~m}$ diameter paraboloidal reflector with focal length-to-diameter ratio of 0.28 , which is detailed in the paper. Residual cooling stresses from the manufacturing process often induce relatively large distortions in thin CFRP structures - potentially reducing their surface accuracy. It is shown by analysis that these distortions are reduced by a factor of 100 in the proposed stiffened reflector.
\end{abstract}

\section{Nomenclature}

$A_{i}$

$D$

E

F

*Lecturer in Structural and Solid Mechanics, 1.tan@ucl.ac.uk ${ }^{\dagger}$ Professor of Structural Engineering, Associate Fellow AIAA, pellegrino@eng.cam.ac.uk

Copyright (C) 2004 by L.T.Tan. Published by the American Institute of Aeronautics and Astronautics, Inc. with permission. $\lambda$ dimensionless parameter in calculation of natural frequency of spherical shells

$\phi \quad$ subtended angle of spherical cap

$\psi \quad$ curvature of reinforcing curve

$\rho$ density

$\sigma_{y} \quad$ ultimate stress

$\sigma_{\text {limit }}$ limit stress

$\sigma_{\max }$ maximum stress in the reflector, excluding slit elements

$\theta \quad$ stiffener angle from vertical axis

CFRP carbon fibre reinforced plastic

rms root mean square

\section{Introduction}

This paper is concerned with deployable reflectors that consist of a thin-walled, parabolic dish made of carbon fibre reinforced plastic (CFRP), which are then elastically folded for launch on a spacecraft. These reflectors are constructed as a single piece, without any joints or hinges, and hence are relatively inexpensive to manufacture. The folding concept, first proposed by Robinson ${ }^{6}$ is both simple and effective: opposite edges of the reflector are pulled towards each other by about half their original distance and are held by tie cables, see Figure 2. The antenna is designed to fit in the normally unused space at the top of the rocket fairing or around the payload, its largest stowed dimension being slightly larger than the deployed diameter. Once in orbit, the tie cables that hold the reflector in its packaged configuration are released by pyrotechnic charges and the reflector deploys dynamically by releasing its stored elastic strain energy.

In order to be folded elastically, a parabolic dish needs to have low stiffness, however in general low stiffness in a structure is accompanied by low shape 


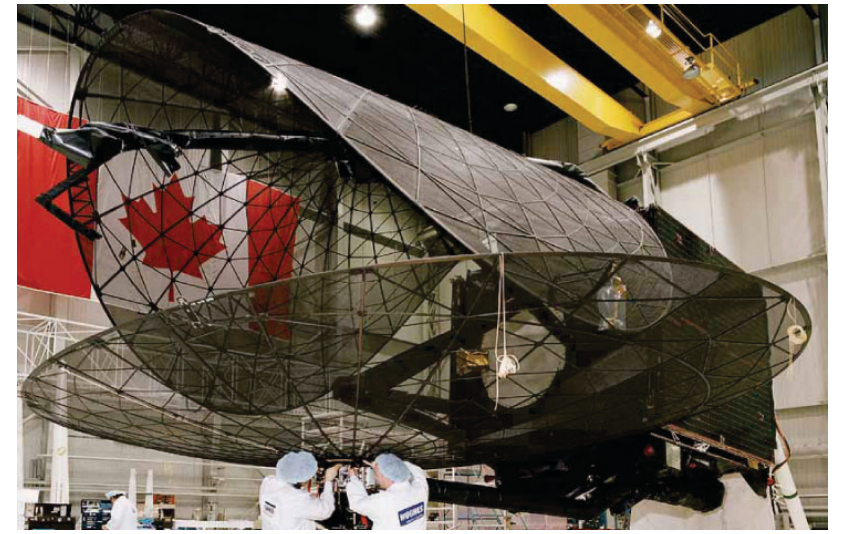

Fig. 1 Spring-Back Reflector in deployed and packaged configurations (Courtesy of the Canadian Space Agency http://www.space.gc.ca.)

accuracy. Furthermore, the manufacturing process of CFRP structures leaves behind residual strains that generate relatively large shape distortions in such highly flexible structures. These distortions are typically of the order of $D / 100$ in an unstiffened thin parabolic dish. We have recently proposed ${ }^{10,11}$ a new concept for this type of reflector structures. Our approach is to stiffen the parabolic dish by attaching a collapsible stiffener around its edge. This stiffener significantly increases the overall stiffness of the dish in the deployed configuration and yet during folding the stiffener "pops" or buckles and thus the structure can still be folded elastically. In our previous paper we demonstrated this approach for small-scale reflectors, and we showed - both by finite-element simulation and on physical models - that order-of-magnitude increases in stiffness could be achieved.

The present paper extends this approach to a fullscale reflector structure. The method for increasing the stiffness of a given reflector while also avoiding excessive strains in the material during folding is briefly reiterated, a reference reflector design is presented and a simple method is used to obtain an estimate of its fundamental natural frequency of vibration. Following this, the reflector design is characterized with extensive FE simulations and the design parameters of the stiffener are optimized. The effects of cooling stresses
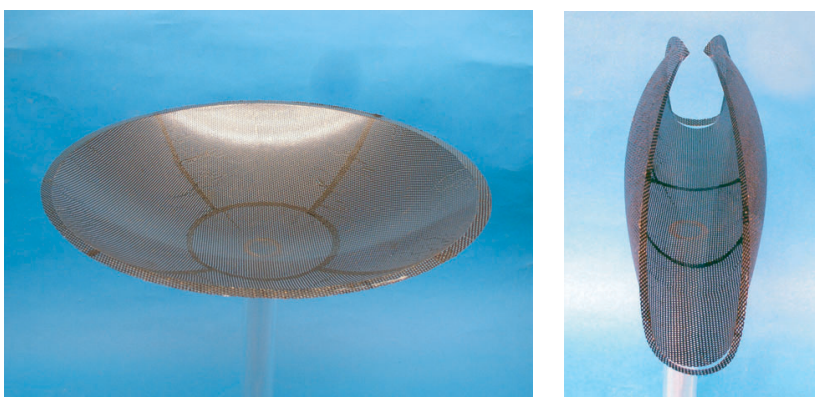

Fig. 2 Small scale model reflector, deployed and folded.

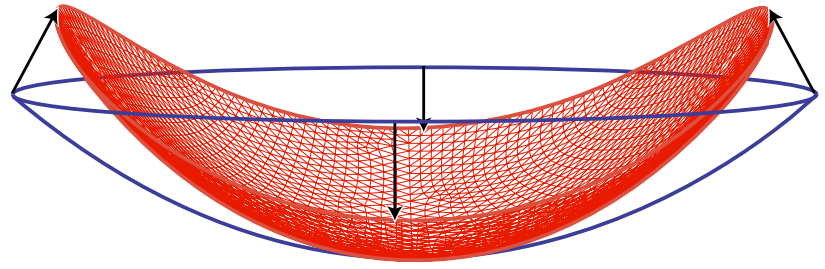

a) Spherical cap

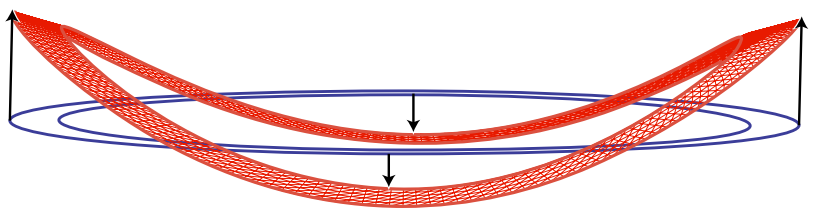

b) Stiffener

Fig. 3 Lowest stiffness, incompatible eigenmodes of a spherical cap and a conical stiffening ring.

on the shape of a stiffened reflector are also investigated and it is shown that a particular stiffener design is capable of reducing manufacturing distortions by two orders of magnitude. The optimization study of the full-scale reflector produced optimal designs which have increases in the stiffness (of the softest mode) of the reflector by 8.5 up to 163.4 -fold, and fundamental natural frequency increases of between 2.6 and 13.6-fold. In all cases a safety margin of at least 2 is maintained on the breaking strength of the material.

\section{Review of Stiffening Scheme}

The stiffening scheme is based on the idea of preventing the unstiffened dish from deforming in its lowest stiffness eigenmode. In the case of an "open cap" shell this eigenmode is the inextensional or first bending mode sketched in Figure 3 (a). Connecting on a second shell in the form of a planar ring, Figure 3 (b), whose lowest stiffness eigenmode is incompatible with that of the first shell has the effect of substantially increasing the stiffness of the open cap shell. It is therefore, a very efficient way of increasing the stiffness of the structure. The exact shape of the second shell is not crucial, hence variations of this method of stiffening would be to connect a torus, part of a conical shell, or just a straight horizontal stiffener. A conical shell will be chosen, for simplicity. However, the problem of attaching a continuous stiffener to a reflector dish, is that it makes the reflector so stiff that it can no longer be folded elastically. In our previous paper ${ }^{11}$ this problem was addressed by introducing four circumferential slits between the stiffener and the rim of the dish, resulting in a structure whose stiffness can be tuned as required. A particular feature that is obtained for sufficiently long slits is that the unsupported lengths of the stiffener are able to buckle while the reflector is being folded, thus decreasing the force required to fold the reflector. A potentially neg- 


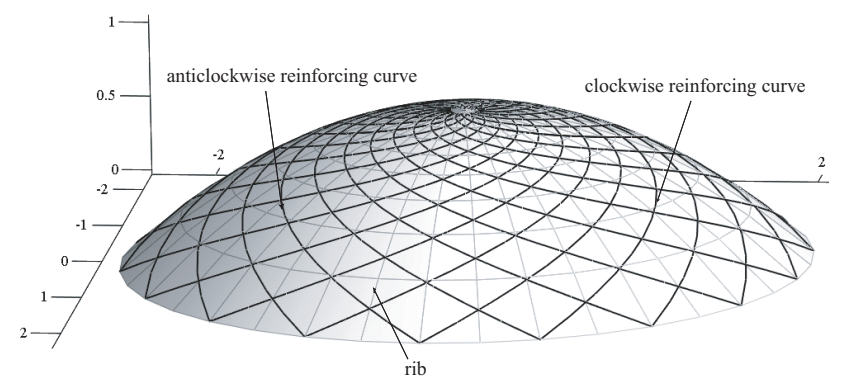

Fig. 4 Reflector geometry.

ative effect of introducing cuts in any structure is that they may result in high stress concentrations. However these can be eliminated by designing out sharp edges and/or by controlling the stiffness distribution near the cuts, see the Stress Concentrations section.

\section{Properties of the Reflector}

The particular structure that will be studied has an aperture diameter of $4.6 \mathrm{~m}$ and a focus to diameter ratio, $F / D=0.28$. It will be assumed that the dish is formed by laying up one or more layers of $0.3 \mathrm{~mm}$ thick triaxially woven CFRP on a paraboloidal mould, thus forming an ultra thin shell (surface thickness, $t_{\text {surf }}=0.3 \mathrm{~mm}$ ) with a series of reinforcements, with a thickness, $t_{\text {rein }}=2.4 \mathrm{~mm}$. The reinforcements consist of a circular edge beam, 36 radial ribs, 18 reinforcing curves spiraling clockwise and 18 more spiralling anticlockwise from the center of the reflector, Figure 4 and Figure 8. All these reinforcements form a system of triangulated segments, similar to those of the Spring-Back Reflector shown in Figure 1.

\section{Material}

The most common triaxial fabric has yarn orientations at $0^{\circ},+60^{\circ},-60^{\circ}$. CFRP made from this fabric has nearly isotropic elastic modulus ${ }^{3,8,12}$ and high in plane shear rigidity. Fujita ${ }^{3}$ provides the following data for CFRP made from SK-802 fabric, manufactured by Sakase Adtech Co. Ltd., Japan, and EPIKOTE 828 epoxy matrix, manufactured by Resolution Performance Products LLC. SK-802 consists of T-300 fibres $\left(E_{\text {fibre }}=230 \mathrm{GPa}\right)$ with a fibre volume fraction of $40 \%$. This triaxially woven fabric has an areal density of $75 \mathrm{~g} / \mathrm{m}^{2}$ and a ply thickness of $0.13 \mathrm{~mm}$, from which we can deduce a fabric density of $0.578 \mathrm{~g} / \mathrm{cm}^{3}$. If we then assume that the epoxy $\left(\rho_{\text {epoxy }}=1.25 \mathrm{~g} / \mathrm{cm}^{3}\right)$ occupies the remaining volume, using the rule of mixtures we obtain $\rho_{\text {composite }}=$ $0.98 \mathrm{~g} / \mathrm{cm}^{3}$. The elastic modulus of this composite was assumed to be $40 \mathrm{GPa}$, and the ultimate stress to be $300 \mathrm{MPa}$.

\section{Simple Frequency Estimates}

An analytical estimate of the fundamental natural frequency of a parabolic dish can be obtained by ap-

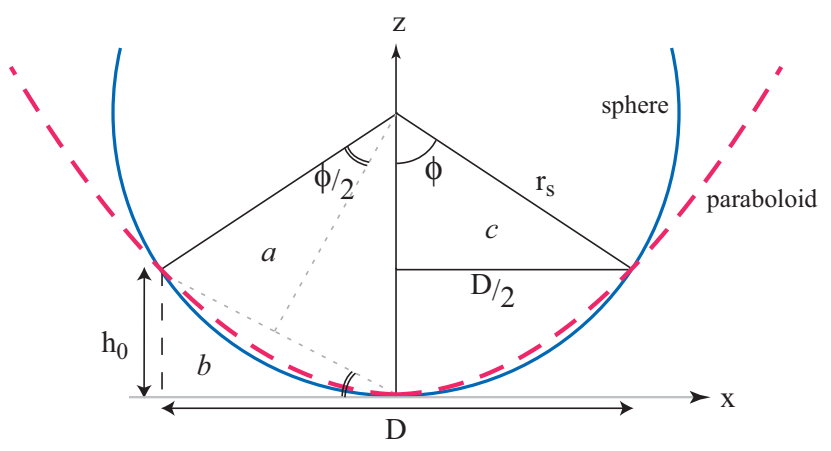

Fig. 5 Approximation of paraboloid with spherical cap.

proximating the dish with a spherical cap of uniform thickness, Figure 5. The spherical equivalent of the reflector described above is found as follows. First, the radius of the sphere and the angle subtended need to be determined. For a parabola with focal length $F$, the height of the $\operatorname{rim}, h_{0}=\frac{D^{2}}{16 F}$. From triangle $a$ in Figure 5,

$$
\sin \frac{\phi}{2}=\frac{\sqrt{\left(\frac{D}{2}\right)^{2}+h_{0}^{2}}}{2 r_{s}}
$$

and from triangle $b$,

$$
\sin \frac{\phi}{2}=\frac{h_{0}}{\sqrt{\left(\frac{D}{2}\right)^{2}+h_{0}^{2}}}
$$

Eliminating $\sin \frac{\phi}{2}$ from Equations (1) and (2) and substituting for $h_{0}$ yields the radius of the sphere

$$
r_{s}=2 F+\frac{D^{2}}{32 F}
$$

Next the subtended angle $\phi$, of the spherical cap needs to be determined. From triangle $c$ in Figure $5, \sin \phi=$ $\frac{D / 2}{r_{s}}$. Therefore substituting for $r_{s}$ gives the subtended angle of the spherical cap

$$
\phi=\sin ^{-1}\left(\frac{D}{4 F+\frac{D^{2}}{16 F}}\right)
$$

In the present case, with $F=1.28 \mathrm{~m}$ and $D=4.57 \mathrm{~m}$, the radius of the equivalent sphere is $r_{s}=3.07 \mathrm{~m}$ and the subtended angle is $\phi=48.11^{\circ}$. An analytical expression for the fundamental natural frequency of vibration of a deep $\left(h_{0} \geq D / 8\right)$, open spherical shell was originally obtained by Rayleigh and is available in Blevins $^{1}$

$$
f=\frac{\lambda}{2 \pi r_{m}} \sqrt{\frac{E}{\rho}}
$$

where

$$
\lambda=\sqrt{\frac{12}{3(1+\nu)}\left(\frac{t}{r_{m}}\right)^{2} \frac{g_{1}}{g_{2}}}
$$


where

$$
\begin{aligned}
& g_{1}=\frac{1}{8}\left[\left(\tan \frac{\phi}{2}\right)^{2}+\left(\tan \frac{\phi}{2}\right)^{4}+\frac{\left(\tan \frac{\phi}{2}\right)^{6}}{3}\right] \\
& g_{2}=\int_{0}^{\phi}\left[(2+\cos \phi)^{2}+2(\sin \phi)^{2}\right]\left(\tan \frac{\phi}{2}\right)^{4} \sin \phi d \phi
\end{aligned}
$$

Here, $t$ is the thickness of the shell, $E$ and $\rho$ are the elastic modulus and the density of the shell. Note that this is the frequency estimate for a spherical cap with uniform thickness. A CFRP spherical cap with an equal mass to the reflector* described earlier would have to have a uniform thickness of $t=0.76 \mathrm{~mm}$. Resulting in $\lambda=7.11 \times 10^{-4}$ and a corresponding fundamental natural frequency of $0.24 \mathrm{~Hz}$

\section{Modelling Details}

\section{Geometry}

The parabolic geometry of the reflector (including the complex layout of the triangular reinforcements) was mathematically described by altering a spherical coordinate system with a constant radius into one which has a radius that varies with the co-latitude angle. In order to achieve this, the paraboloid was inverted and translated vertically so that the rim of the reflector lies on the $x-y$ plane, Figure 6 . This results in the following equation for the paraboloid

$$
z=-\frac{\left(x^{2}+y^{2}\right)}{4 F}+h_{0}
$$

where the rim height $h_{0}=1.02 \mathrm{~m}$, occurs at the co-latitude $\alpha=90^{\circ}$. The variation of the paraboloidal radius as a function of the co-latitude, $\alpha$, can be determined by noting that for points on the $x$ - $z$ plane, $z=x / \tan \alpha$. Equating this to Equation (7), and noting that $y=0$ in the $x-z$ plane gives,

$$
\frac{x}{\tan \alpha}=\frac{-x^{2}}{4 F}+h_{0}
$$

Solving for $x$ results in

$$
x(\alpha)=\frac{-2 F+2 \sqrt{F^{2}+h_{0} F \tan ^{2} \alpha}}{\tan \alpha}
$$

Then from the geometry in Figure 6

$$
\begin{aligned}
r(\alpha) & =\sqrt{x^{2}(\alpha)+z^{2}(\alpha)} \\
& =x(\alpha) \sqrt{1+\frac{1}{\tan ^{2} \alpha}}
\end{aligned}
$$

Hence the $x, y, z$ coordinates of the paraboloid can be described by $r(\alpha) \sin \alpha \cos \beta, r(\alpha) \sin \alpha \sin \beta$, and $r(\alpha) \cos \alpha$ respectively. In order to mathematically describe the geometry of the spiralling curves, it is first assumed that they have a constant curvature, $\psi$, with respect to the longitude, $\beta$ and the co-latitude, $\alpha$. Hence, in a plot of $\beta$ versus $\alpha$, these curves would be represented by straight lines with a gradient of $\tan \psi$, or mathematically by

$$
\beta(\alpha)=\alpha \tan \psi-\alpha_{0} \tan \psi
$$

*this is the reflector with reinforcements described in the Properties of Reflector section.

${ }^{\dagger}$ a spherical shell with uniform thickness, $t=t_{\text {rein }}=2.4 \mathrm{~mm}$ would have a fundamental natural frequency of $0.74 \mathrm{~Hz}$

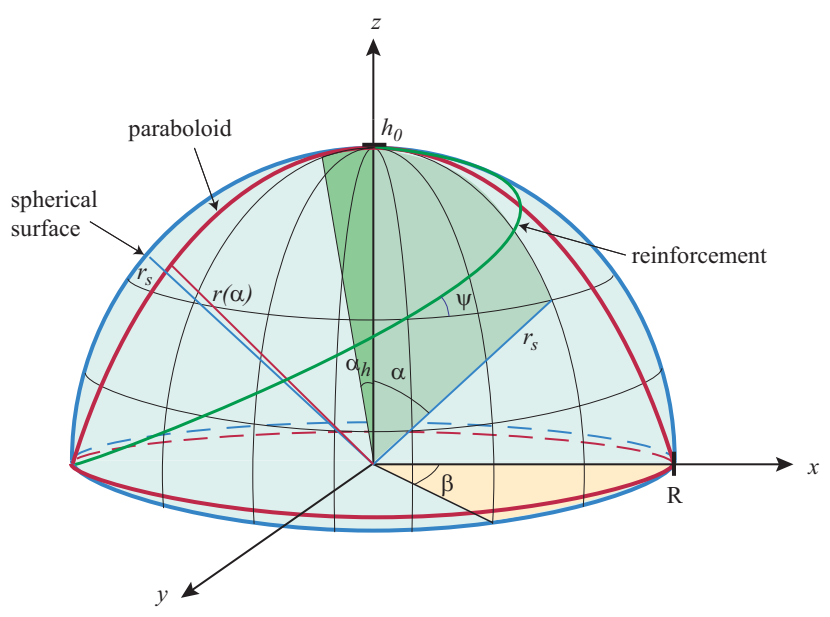

Fig. 6 Spherical coordinate system with paraboloid and reinforcement.

again $\psi$ is the angle the reinforcing curve makes with any latitude of the surface and is representative of the curvature of the curve, while the term $\alpha_{0} \tan \psi$ is the $\beta$-axis intercept. $\alpha_{0}$ represents a point near the apex of the surface, at which the reinforcements start and is chosen by the designer, subject to the condition that $\alpha_{0} \neq 0$. For the purposes of the current reflector, the reinforcements were assumed to start at about $2 \%$ of the diameter, i.e. at a horizontal distance of $x \approx 90 \mathrm{~mm}$ from the center of the dish. Substituting this value into Equation (7), we obtain a value of $z=1018 \mathrm{~mm}$ and then noting that $\tan \alpha_{0}=\frac{x}{z}$, $\alpha_{0}$ becomes $5.1^{\circ}$. Once the value of $\alpha_{0}$ has been defined, the curvature $\psi$ can be determined by observing that the reinforcing curve will intersect alternate ribs at the rim of the dish, Figure 4 . If we consider the $x-z$ plane which only has ribs at $\beta=0^{\circ}$ and $180^{\circ}$ or $\pi$ and then impose the boundary condition that at the rim, $\alpha=90^{\circ}$ or $\frac{\pi}{2}$, Equation (10) results in

$$
\begin{aligned}
& \pi=\frac{\pi}{2} \tan \psi-\alpha_{0} \tan \psi \\
& \psi=\arctan \left(\frac{2 \pi}{\pi-2 \alpha_{0}}\right)
\end{aligned}
$$

which gives $\psi=64.75^{\circ}$. Therefore the governing equations for the two families of curves (spiralling clockwise and anticlockwise, i.e. $\pm \psi$ ) are

$$
\beta\left(\alpha, \beta_{c}\right)=\alpha \tan ( \pm \psi)+\alpha_{0} \tan ( \pm \psi)+\beta_{c}
$$

$\beta_{c}$ is indicative of the longitudinal starting point of each curve - there are 18 curves in each family and hence $\beta_{c}=$ $n \times 20^{\circ}$ where $n=1,2, \ldots 18$.

Equation (11) results in the following cartesian coordinates for the reinforcing curves:

$$
\begin{aligned}
& x_{c}=r(\alpha) \sin \alpha \cos \left(\beta\left(\alpha, \beta_{c}\right)\right) \\
& y_{c}=r(\alpha) \sin \alpha \sin \left(\beta\left(\alpha, \beta_{c}\right)\right) \\
& z_{c}=r(\alpha) \cos \alpha
\end{aligned}
$$

\section{Computational Details}

All reinforcements i.e. the reinforcing curves, ribs and edge beam were modelled by means of 3-noded beam elements (element B32), whereas all other surface elements 
were shell elements (element S3R). The beam normals were defined by specifying the directional cosines for all the nodes. All simulations were performed with the ABAQUS finite element package ${ }^{4}$ and consisted of two main steps, the first being an eigenvalue extraction to establish the fundamental frequency of the deployed configuration, and the second step models the packaging process. This second step is a geometrically non-linear static analysis which imposes a prescribed displacement between two diametrically opposite nodes on the rim of the dish, i.e. the edges of the reflector are brought together by a distance $D / 2$. The force-displacement behaviour during packaging is then analysed to give the initial stiffness of the reflector, $k$ and the maximum packaging force, $F_{\max }$.

\section{Optimization}

An automatic optimization scheme was devised to find designs that maximize the deployed stiffness of the reflector - assumed to be proportional to the fundamental natural frequency of the deployed configuration, $f$ - subject to a limit on the maximum stress in the packaged configuration, $\sigma_{\max }$. The objective function which was minimized is

$$
\begin{array}{ll}
\text { if } \sigma_{\text {max }} \leq \sigma_{\text {limit }} & \\
\text { else } & F_{o b j}=-f \\
& F_{o b j}=-f+\left(\sigma_{\text {max }}-\sigma_{\text {limit }}\right)^{2}
\end{array}
$$

This is to penalise solutions which exceed the limit stress, $\sigma_{\text {limit }}$. Limits on the maximum stress were set at $75 \mathrm{MPa}$, $100 \mathrm{MPa}, 125 \mathrm{MPa}$ and $150 \mathrm{MPa}$, for different optimization runs, corresponding to safety factors of roughly 4,3 , 2.5 and 2 on the material failure strength. It needs to be noted that $\sigma_{\max }$ represents the highest stress in the reflector, excluding the elements at the end of the slits. This omission will be justified in the next section. The optimization procedure was implemented via a $\mathrm{C}$-subroutine that implements the Hooke and Jeeves method ${ }^{5}$ and links this to the ABAQUS finite element package for each evaluation of the objective function. The optimization process also requires the automatic creation and meshing of the stiffening system as the different parameters are varied. The parameters to be optimized, see Figure 7, are:

- $\theta$ - stiffener angle from the vertical

- $w$ - stiffener width

- $t_{s}$ - stiffener thickness

- $\eta$ - hinge slit angle

- $\gamma$ - load slit angle

Knowledge and experience gained from the small scale reflector optimization studies, ${ }^{10}$ allowed us to set tighter limits on the variables and hence, reduce the search space. The limits are:

$$
\begin{aligned}
80^{\circ} & \leq \theta \\
0.005 D & \leq w \leq 100^{\circ} \\
2^{\circ} & \leq \gamma, \eta \leq 0.05 D \\
0.5 \mathrm{~mm} & \leq t_{s} \leq 2 \mathrm{~mm}
\end{aligned}
$$

The slits are modelled by generating a ring of narrow rectangular slit elements between the inner edge of the stiffener

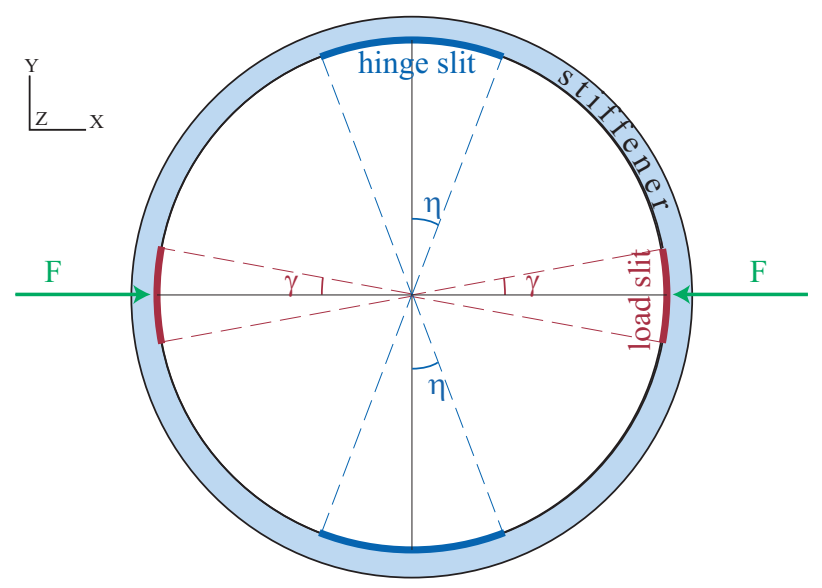

a) Plan view

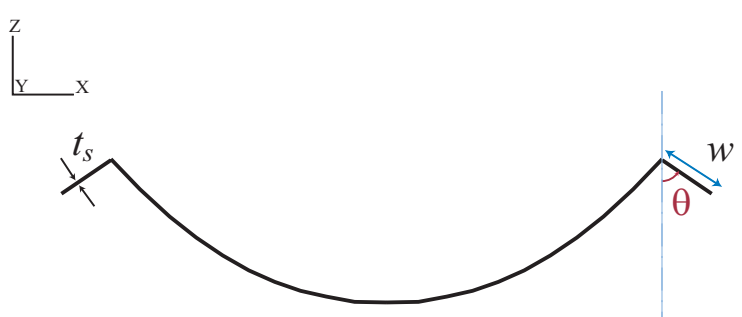

b) Side view

Fig. 7 Parameters to be optimized.

and the rim of the parabolic reflector, Figure 8. The rectangular elements immediately adjacent to the slit (end of slit elements) are allowed to vary in width, the end of slit nodes are then linearly constrained to the rest of the structure. This capability, coupled with a simple routine which calculates the coordinates of the end of the slit, then allows the slit length to be altered continuously. Hence, in reality the slit is actually the void which is not filled up with elements.

This method of modelling the slits (Model SE) was compared to model where the slits were formed by leaving the relevant edge nodes of the stiffener and the dish unconnected (Model SU). The respective masses, $m$, frequencies, $f$, and initial stiffnesses, $k$, are given in Table 1 . These models have identical configurations of $\theta=90^{\circ}, \gamma=2^{\circ}, \eta=$ $12^{\circ}$ and a stiffener thickness, $t_{s}=1.524 \mathrm{~mm}$. The difference between the two models is that in Model SE, the slits are modelled as voids and hence, both the mass and the stiffness are lower. The fundamental natural frequency, $f$, is also affected. A model with lower stiffness will also require less force, $F_{\max }$, to fold it and will undergo a lower maximum stress, $\sigma_{\max }$, when fully packaged. As expected, the larger differences occur during packaging $\left(k, F_{\max }\right.$ and $\left.\sigma_{\max }\right)$, with the largest difference of $6.64 \%$ occurring at $F_{\max }$, the force at which the snap occurs i.e. the stiffener starts to buckle. In practice, however, the slits would have to be of finite width. This also avoids interference during folding. Hence the technique of modelling the slits as voids is the more realistic one. 


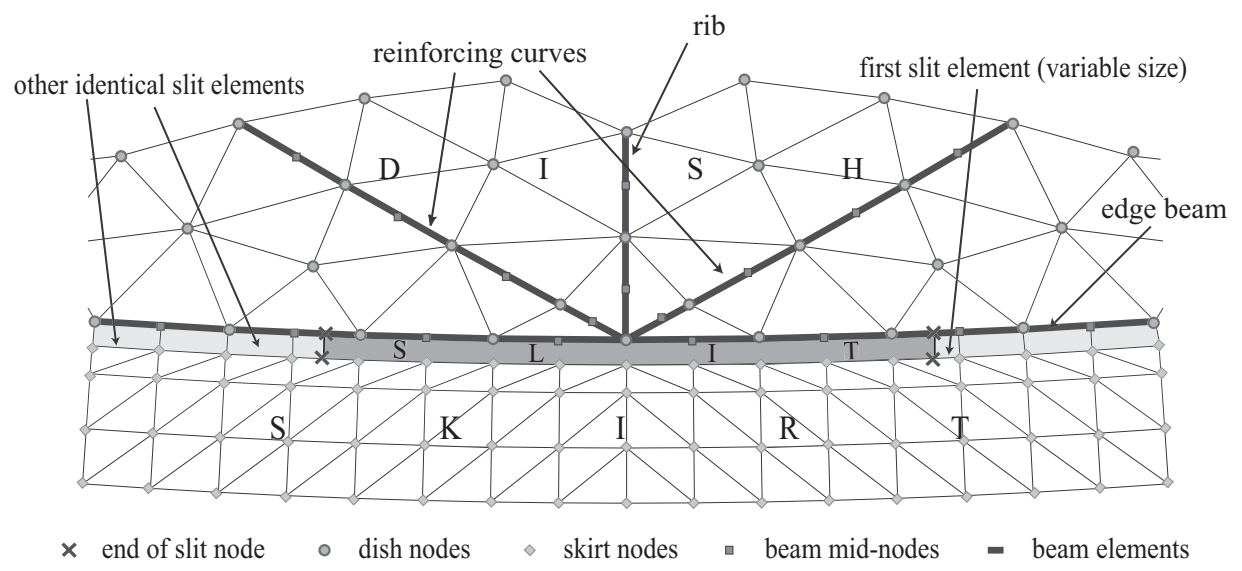

Fig. 8 Schematic of automatically generated mesh.

\begin{tabular}{lccc}
\hline \hline & $S E$ & $S U$ & \% difference \\
\hline$m(\mathrm{~kg})$ & 17.89 & 17.90 & 0.05 \\
$k(\mathrm{~N} / \mathrm{mm})$ & 4.56 & 4.86 & 6.19 \\
$f(\mathrm{~Hz})$ & 6.45 & 6.53 & 1.23 \\
$F_{\max }(\mathrm{N})$ & 19.55 & 20.94 & 6.64 \\
$\sigma_{\max }(\mathrm{MPa})$ & 196.0 & 204.9 & 4.34 \\
\hline \hline
\end{tabular}

Table 1 Comparison of two slit models, SE has rectangular slit elements, SU has unconnected dish and stiffener nodes. Both models have identical slit angles, and stiffener width and angle.

\section{Stress Concentrations}

The tip of the slit presents one of the biggest problems for the design of the stiffening system as it induces a significant stress concentration. This concentration can be reduced by drilling a hole at both ends of the slits - thus rounding off any sharp corners, and consequently reducing any stress concentrations. However the edge beam that forms the edge of the dish should not be drilled through. A method of preventing crack growth in solids is to build in softer, tougher material near the crack tip, i.e. a material with a lower Young's Modulus and/or reduced thickness. In order to implement this change, the mesh of the region surrounding the stress concentration had to be refined.

\section{Refined Mesh and Submodelling}

The mesh near the tip of the slit was refined to an average density of 27 elements $/ \mathrm{cm}^{2}$, i.e. around 770 elements in a $30 \mathrm{~mm}$ radius, made up of concentric circles of elements centered at the tip of the slit, Figure 9(b) and Figure 10. The rest of the reflector mesh remained unaltered. The area of mesh refinement is less than $0.07 \%$ of the total area of the reflector, hence rather than running a simulation of the entire reflector to capture the behaviour of the small region around the slit, the submodelling procedure in ABAQUS was used. This procedure allows the user to model only a certain part of the larger global model. The user defines the submodel in a separate input file and decides which nodes of the submodel are to be driven by results from the global model. ABAQUS then searches the global model for these nodes or interpolates results for these nodes based on a set of user-defined nodes or ele- ments. ABAQUS also provides the capability of selecting the variables which are used to drive the analysis - for our purposes the displacement variables were selected in order to model the packaging of the reflector. By selecting a large enough submodel, Figure 9(a), the overall effect of changing the thickness and material properties of the end of slit region, inset of Figure 9(a), is minimized. Another considerable advantage of the submodelling routine is the reduced run times (by a factor of 10). To maintain the generality of this study, a general non-optimal configuration with $\theta=90^{\circ}, w=120 \mathrm{~mm}, \eta=16^{\circ}, \gamma=12^{\circ}$, was chosen, resulting in a total mass of $17.98 \mathrm{~kg}$ and a fundamental frequency of $5.53 \mathrm{~Hz}$. The maximum Von Mises stress in this model is $175 \mathrm{MPa}$, occurring in the rectangular slit element at the end of the hinge slit. Neglecting this element, the next highest stress is $120 \mathrm{MPa}$ and occurs in one of the triangular stiffener elements near the edge of the hinge slit. Once the submodel mesh is refined (for generality the slits were modelled as unconnected portions of the stiffener and rim, resulting in the worst case scenario of an infinitesimal slit tip radius), the stress at the slit tip increases to $432 \mathrm{MPa}$.

\section{Stress Reduction}

The stress concentrations at the slit tips were lowered by reducing the stiffness and thickness of the stiffener elements immediately surrounding the tip and then gradually ramping these values back up towards the original stiffener thickness. Figure 10(a) shows the thickness of the various elements and their respective material properties. Two flexible materials were chosen, one with the same density of the CFRP used in the rest of the reflector, but three quarters the stiffness, $E=30 \mathrm{GPa}$, which we will call epoxy 2 , while the other is just the epoxy by itself (epoxy 1 ). It is worth noting that the 'dish' part of the reflector does not experience very high stresses, as the edge beam acts to redistribute the stresses to the rest of the dish, hence most of the high stresses are concentrated in the stiffener region. As a result, the thickness and Young's Modulus of only one element on the dish portion had to be altered. This is a great advantage, as if any permanent deformation were to occur, it would be in the stiffener, and thus, the actual working part of the reflector would be left undamaged. By surrounding the slit with these more flexible materials, the maximum stress in the reflector can be reduced by 3.3 


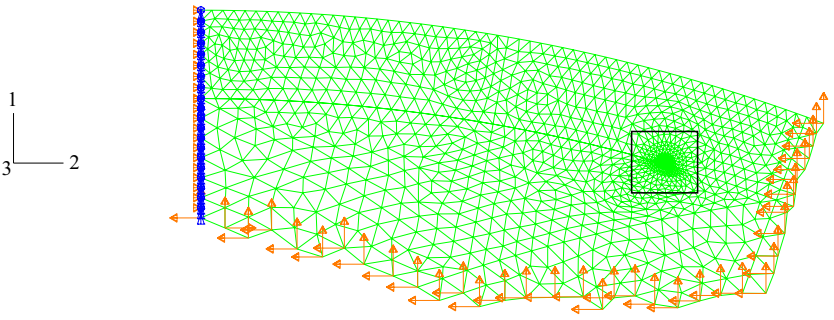

a) Boundary conditions of the submodel

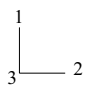

b) Refined mesh region around slit tip

Fig. 9 Submodel mesh.

times from $432 \mathrm{MPa}$ to $132.6 \mathrm{MPa}$. This value is only $10 \%$ higher than the highest stress present in the reflector, after the stresses in the slit elements have been neglected, i.e. $120 \mathrm{MPa}$. Some simple further redesign would reduce this value even more. Even further reduction could also be gained from rounding off the sharp edge at the tip of the slit. It is worth noting that the models considered for this stress reduction process do not have the rectangular slit elements, in Figure 8, which were used in the optimization process, but instead the slits were modelled as unconnected portions of the stiffener and dish. In practice, having a finite slit width is advantageous as the slit tip would then have a finite radius - resulting in lower stresses at the tip. As it currently stands, the total region of altered material properties, i.e. 8 slit tip regions, is less than $0.55 \%$ of the total area of the reflector. Hence, by altering a negligible amount of the reflector surface, the stresses at the tip of the slits can be reduced to a level close to that experienced by the rest of the reflector. It has been shown that the stress in the tip region can be reduced by a factor of nearly 3.3 compared to a model with a similar mesh, or a factor of nearly 1.3 when compared to the coarser mesh model used in the optimization runs. Therefore justifying the procedure adopted in the optimization study, of neglecting the high stresses occurring in the slit elements.

\section{Distortion and Shape Accuracy}

The manufacturing process of ultra thin CFRP structures leaves behind residual stresses that can produce large shape distortions. To capture the effect of the stiffener, a comparison is made with a reflector without the stiffener (unstiffened reflector). Based on the assumption that the stiffened and unstiffened reflectors will experience similar amounts of residual cooling stresses, these distortions were modelled by applying the equivalent forces required to cause the magnitudes of distortion that are typically observed in practice. A distortion amplitude of $5.9 \mathrm{~mm}$ was assumed (for a dish without a stiffener), which manifests as

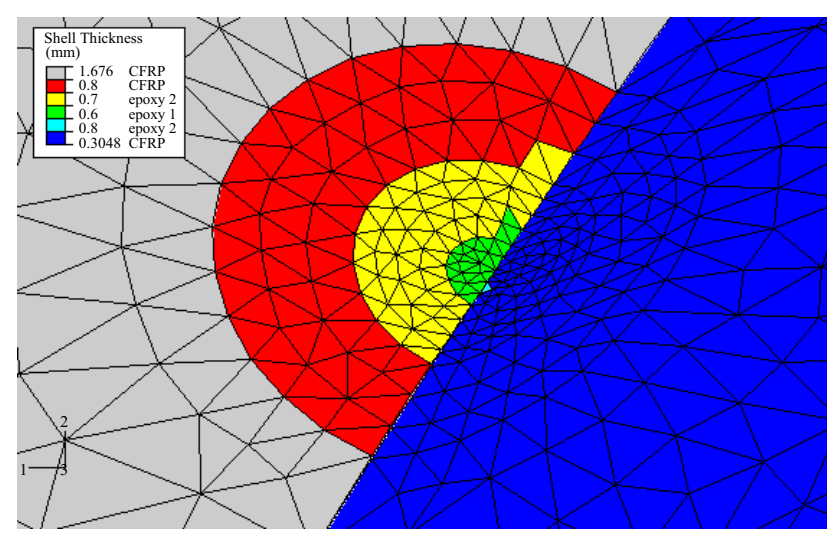

a) Shell element thicknesses of the slit tip region.

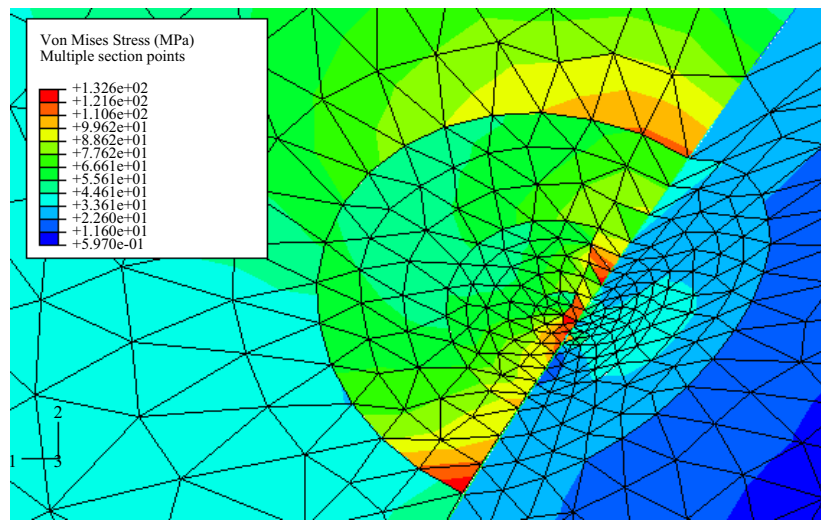

b) Von Mises stress distribution of this modified slit tip region. The maximum stress has been reduced from $432 \mathrm{MPa}$ to 132.6 $\mathrm{MPa}$

Fig. 10 Stress reduction of slit tip region.

an ovalization of the dish, Figure 11. This deformation can be produced in the unstiffened dish by means of two equal and diametrically opposite forces of only $0.06 \mathrm{~N}$. Considering the aperture of the reflector, $D=4.6 \mathrm{~m}$, these forces which are equivalent to masses of 6 grammes are minuscule. These forces were then imposed on a stiffened reflector with a configuration of $w=120 \mathrm{~mm}, \theta=90^{\circ}, \eta=16^{\circ}, \gamma=12^{\circ}$ and $t_{s}=1.676 \mathrm{~mm}$. This resulted in a maximum distortion of $0.057 \mathrm{~mm}$, Figure 12, while the maximum distortion in the $z$-direction is $0.046 \mathrm{~mm}$ - roughly $1 / 100^{\text {th }}$ and $1 / 120^{\text {th }}$ of the distortions experienced by the unstiffened reflector.

\section{Shape Accuracy}

The root mean square (rms) surface accuracy of the distorted reflectors was determined by using the least squares $\operatorname{method}^{9}$ to solve for the best fit paraboloid, hence minimizing the distance between the surface of the paraboloid and the nodes of the distorted reflector. The axial rms error is then

$$
\delta_{z}=\sqrt{\frac{\sum_{i=1}^{n} A_{i}\left(\hat{z}_{i}-z_{i}\right)^{2}}{\sum_{i=1}^{n} A_{i}}}
$$

where $A_{i}$ is the surface area associated with the $i^{\text {th }}$ node, $\hat{z_{i}}$ is the $z$ coordinate of the best fit paraboloid and $n$ the total number of nodes, for this case 5323 .

The effective rms error $^{7}$ is related to the axial rms error, 


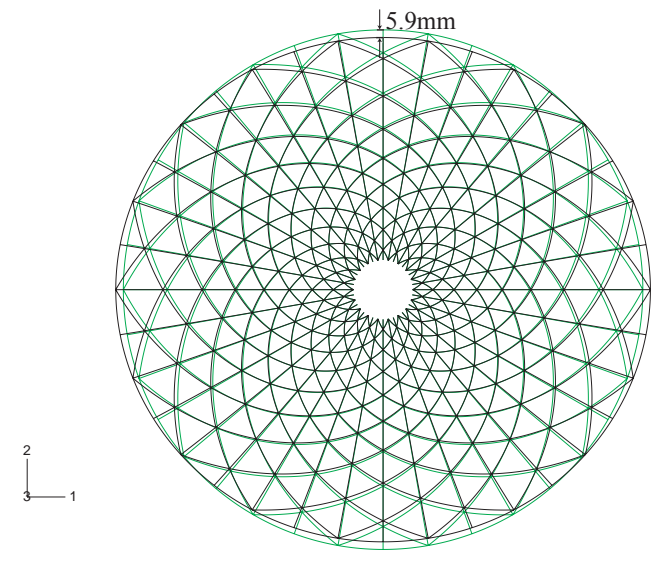

a) Plan view

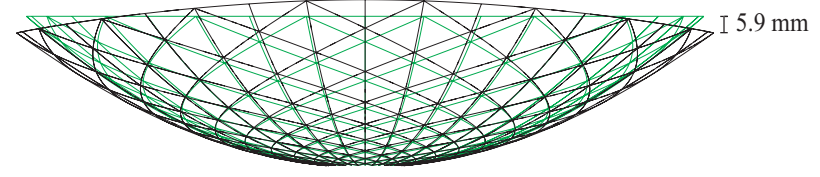

b) Side elevation

Fig. 11 Distortion of reflector without stiffener (magnified by 20). The deformed shape is drawn in black.

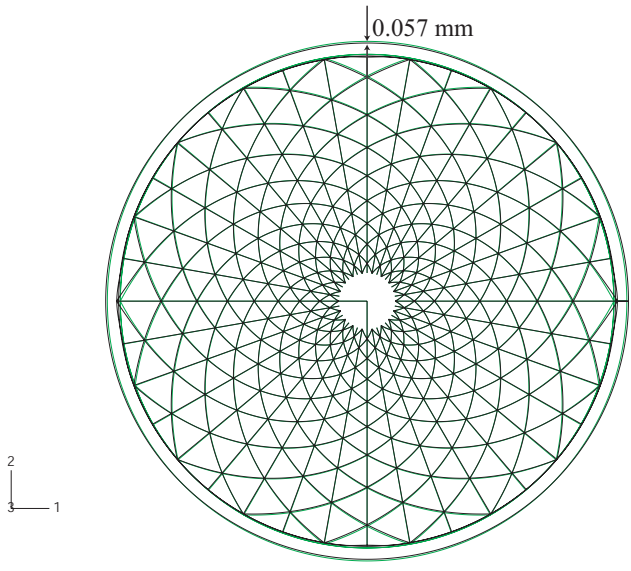

a) Plan view

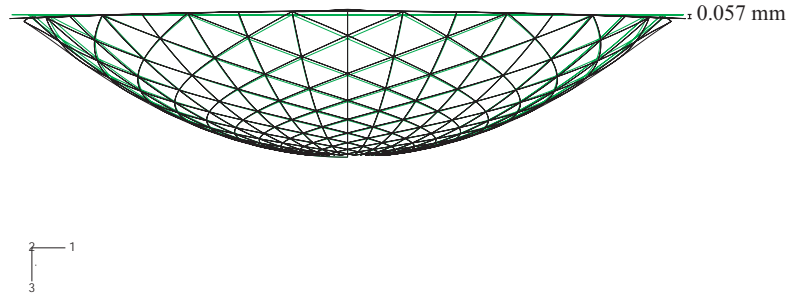

b) Side elevation

Fig. 12 Distortion of reflector with stiffener (magnified by 1000). The deformed shape is drawn in black.

by $\delta_{e}=\delta_{z} /\left(1+(D / 4 F)^{2}\right)$, resulting in an effective rms error of

$$
\delta_{e}=\frac{1}{1+\left(\frac{D}{4 F}\right)^{2}} \sqrt{\frac{\sum_{i=1}^{n} A_{i}\left(\hat{z}_{i}-z_{i}\right)^{2}}{\sum_{i=1}^{n} A_{i}}}
$$

The rms error of the unstiffened reflector with its distortions due to residual strains was found to be $1.84 \mathrm{~mm}$. The addition of the stiffener reduces this error to $0.29 \mathrm{~mm}$. A reduction by a factor of 6.4 compared to the original reflector. However, it needs to be noted that the surface accuracy of the "perfect" reflector i.e. the undistorted FE mesh, is already $0.27 \mathrm{~mm}$. This is basically a measure of how well the triangular shell elements approximate the given parabolic shape. The geometry was created by importing the coordinates of 200 points along a rib and a reinforcing curve into the PATRAN ${ }^{2}$ preprocessor. Each triangulated segment was then created by using the relevant parts of the ribs and reinforcing curves as edges. These triangular surfaces were then automatically meshed using the "paver" option. This is an automatic technique and the user has no control over the shape functions. The maximum $z$ deviation of the nodes in the undistorted FE mesh from the original paraboloid was found to occur in the middle of the largest triangulated segments i.e. the segments nearest the rim and hence the ones with the highest curvature. Hence the most probable source of this error is

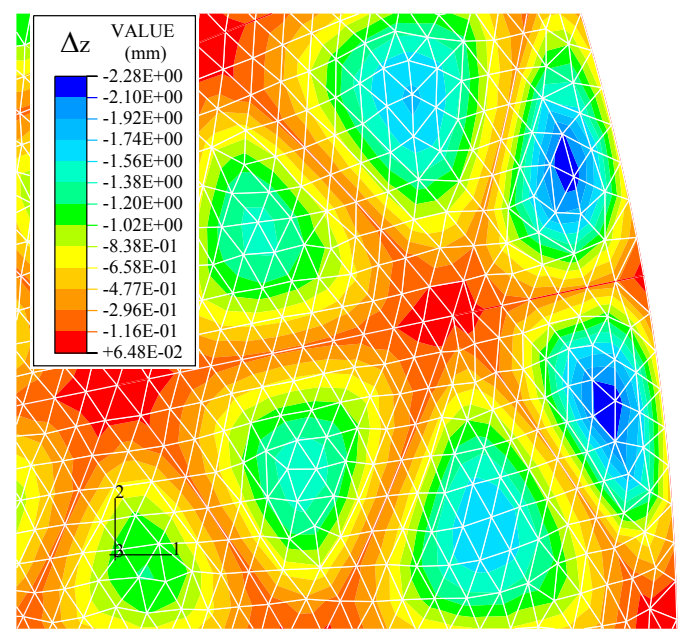

Fig. 13 Contour plot of $z$ deviation between undeformed FE mesh and actual paraboloid, showing regions of maximum deviation. 


\begin{tabular}{lccccccccccc}
\hline \hline Config & $\begin{array}{c}\theta \\
(\mathrm{deg})\end{array}$ & $\begin{array}{c}\eta \\
(\mathrm{deg})\end{array}$ & $\begin{array}{c}\gamma \\
(\mathrm{deg})\end{array}$ & $\begin{array}{c}w \\
(\mathrm{~mm})\end{array}$ & $\begin{array}{c}t_{s} \\
(\mathrm{~mm})\end{array}$ & $f$ & $f / f_{o}$ & $\sigma_{\max }$ & $\sigma_{y} / \sigma_{\max }$ & $k / k_{o}$ & $m / m_{o}$ \\
\hline $\mathrm{O}$ & - & - & - & - & - & 0.53 & 1.0 & 46 & 6.5 & 1.0 & 1.00 \\
$\mathrm{I}$ & 90 & 18 & 12 & 55 & 0.5 & 1.4 & 2.6 & 75 & 4.0 & 8.5 & 1.02 \\
$\mathrm{II}$ & 91 & 16 & 8 & 80 & 1.4 & 3.6 & 6.8 & 100 & 3.0 & 47.5 & 1.10 \\
$\mathrm{III}$ & 90 & 14 & 7 & 100 & 1.5 & 4.8 & 9.1 & 124 & 2.4 & 86.7 & 1.14 \\
$\mathrm{IV}$ & 90 & 12 & 8 & 156 & 1.5 & 7.2 & 13.6 & 151 & 2.0 & 163.7 & 1.23 \\
\hline \hline
\end{tabular}

Table 2 Results of optimization runs. Config O is a dish without stiffening skirt and has $f_{o}=0.529 \mathrm{~Hz}$, mass, $m_{o}=14.66 \mathrm{~kg}$ and an initial stiffness, $k_{o}=0.05 \mathrm{~N} / \mathrm{mm}$.

the inaccuracies caused by creating and meshing the model in PATRAN. The simplest way of accounting for this modelling error and thus determine the absolute value of the rms error in the distorted reflectors, is to subtract the rms value of the undeformed mesh from any subsequent values. Hence, it is concluded that the distorted reflector without the stiffener has an absolute rms error of $1.57 \mathrm{~mm}$ while the addition of the stiffener reduces this to $0.02 \mathrm{~mm}$.

\section{Results and Discussion}

It is again useful to compare the reflector with a dish without the collapsible stiffener, Configuration O. Depending on the stress limit set, optimized designs were found to have initial stiffnesses of up to 164 times higher, and deployed frequencies up to 14 times higher than the unstiffened dish. A set of key results are listed in Table 2 . The extra mass associated with the stiffener varies from as little as $2 \%$ up to $23 \%$. Although pointing the stiffener

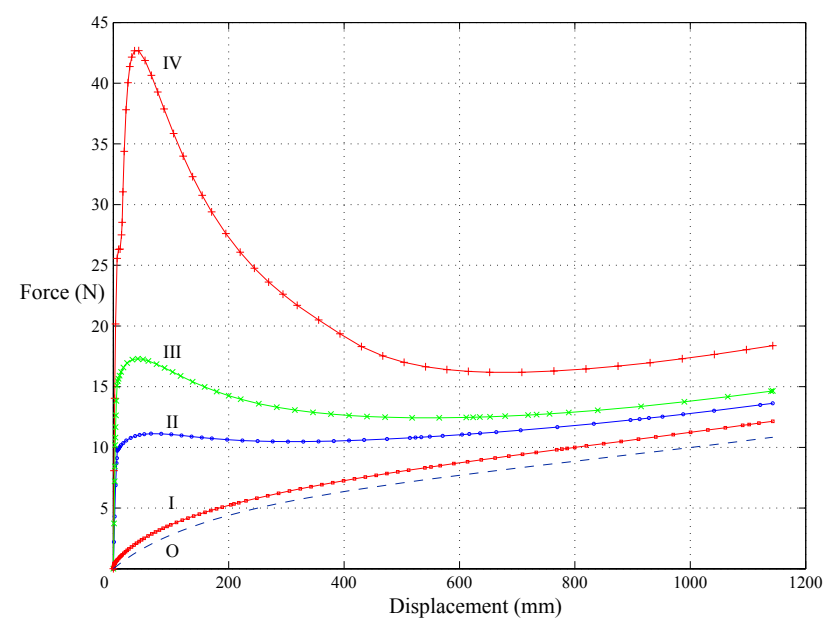

Fig. 14 Force-displacement behaviour of Configurations I-IV and $\mathrm{O}$.

below the level of the rim of the dish is a more efficient way of increasing the stiffness against first-mode deformation, it also produces higher stress levels during folding, hence all the optimized configurations have stiffener angles, $\theta \approx 90^{\circ}$ as this tends to be the best trade off between high stiffness and lower stress levels. It is worth noting that all the stiffness increases have been achieved within acceptable stress limits e.g. even the configuration with the highest stiffness, Configuration IV has a safety factor of 2 on the material failure strength. Figure 14 shows graphs of the force-displacement behaviour during the packaging process of the configurations presented in Table 2 . The majority of the optimized configurations show a snapping behaviour, wherein a large force increment is required initially to fold the reflector, but this suddenly reduces to magnitudes which are comparable to that of the unstiffened reflector. This snapping behaviour corresponds to the initiation of buckling of the unsupported lengths of stiffener, which has the effect of significantly lowering the forces needed to package the reflector. It should be noted that non-optimized structures tend not to show this type of behaviour. Furthermore, the addition of the stiffening scheme was found to reduce manufacturing distortions from $5.9 \mathrm{~mm}$ for the unstiffened reflector to $0.057 \mathrm{~mm}$, nearly $1 / 100^{t h}$ the magnitude, resulting in an estimated rms surface accuracy of about $0.02 \mathrm{~mm}$.

\section{Conclusion}

It has been shown that an ultra-thin CFRP reflector with an aperture of $4.6 \mathrm{~m}$ and focal length of $1.28 \mathrm{~m}$, which has a mass of $14.66 \mathrm{~kg}$, can be stiffened by the addition of a collapsible edge stiffener. Several designs for this stiffener have been presented in Table 2. The lightest of these designs would increase the mass of the reflector by $2 \%$, and would increase the deployed softest-mode stiffness by a factor 8.5 and the fundamental natural frequency by a factor of 2.6. The heaviest design would increase the mass by $23 \%$, and increase the stiffness 164 times and the fundamental frequency 13.6 times. These increases in stiffness can be achieved within a safety factor of 4 and 2 respectively on the breaking strength of the material. Furthermore, the manufacturing distortions are now nearly insignificant, resulting in a much more accurate reflector and hence potentially an increased operating range.

\section{Acknowledgements}

We thank Professor C.R. Calladine for many helpful suggestions and Dr A. Britto for advice on computational work.

\section{References}

${ }^{1}$ Blevins, R. D. Formulas for Natural Frequency and Mode Shapes. Krieger Publishing Company, ch. 12, pp. 330-334.

${ }^{2}$ The MACNEAL-SChWENDLER Corporation $M S C / P A T R A N$ Version 8.5. MSC Software, Los Angeles, California, USA, 1999.

${ }^{3}$ Fujita, A., Hamada, H., and Maekawa, Z. Tensile properties of carbon fiber triaxial woven fabric composites. Journal of Composite Materials 27, 15 (1993), 1428-1442.

${ }^{4}$ Hibisit, K., And Sorenson. ABAQUS Standard Users Manual Version 6.1. Hibbitt, Karlsson and Sorenson, Hibbitt, 
Karlsson \& Sorenson, 1080 Main Street Pawtucket, Rhode Island 02860-4847, USA, 1998.

${ }^{5}$ Hooke, R., And Jeeves, T. A. Direct search solution of numerical and statistical problems. Journal of $A C M 8$ (April 1961), 212-229.

${ }^{6}$ Robinson, S. A. Simplified spacecraft antenna reflector for stowage in confined envelopes. Publication number: 0534110A1, 31 March 1993. European Patent Application filed by Hughes Aircraft Company.

${ }^{7}$ Ruze, J. Antenna tolerance theory - a review. IEEE 54, 4 (April 1966), 633-640.

${ }^{8}$ Skelton, J. Triaxially woven fabrics: Their structure and properties. Textile Research Journal 41, 8 (August 1971), 637647.

${ }^{9}$ Strang, G. Linear algebra and its applications, third ed. Harcourt Brace Jovanovich College Publishers, San Diego, U.S.A., 1988.

${ }^{10}$ TAN, L. T. Thin-Walled Elastically Foldable Reflector Structures. PhD thesis, Department of Engineering, University of Cambridge, Cambridge, CB2 1PZ, UK, December 2002.

${ }^{11}$ Tan, L. T., And Pellegrino, S. Stiffness design for spring back reflectors. In 43rd AIAA/ASME/ASCE/AHS/ASC Structures, Structural Dynamics and Materials Conference (Denver Colorado, 22-25 April 2002). AIAA 2002-1498.

12 Watanabe, A., Tadokoro, H., and Arai, Y. Tensile properties of twf reinforced cf/ep single ply composites. In Materials and Process Affordability, Keys to the Future (June 1998), H. S. Kliger, Ed., vol. 43 of 43rd International SAMPE Symposium and Exhibition, Society of Advancement of Material and Process Engineering (SAMPE), SAMPE, pp. 1874-1882. 